\title{
Bulky Uterus and Multiparity are Important Contributing Factors for Dysfunctional Uterine Bleeding among Bangladeshi Women
}

\author{
Nahid Sultana ${ }^{1}$, Nasima Akhter ${ }^{2}$, TA Chowdhury ${ }^{3}$ \\ Received: May 6, 2015 Accepted: December 19, 2015 \\ doi: http://dx.doi.og/10.3329/jemc.v6i1.26376
}

\begin{abstract}
Background: Dysfunctional uterine bleeding $(D U B)$ is irregular uterine bleeding that occurs in the absence of recognizable pelvic pathology, general medical disease, or pregnancy. It reflects a disruption in the normal cyclic pattern of ovulatory hormonal stimulation to the endometrial lining. About 1-2\% of women with improperly managed anovulatory bleeding eventually may develop endometrial cancer but determinants behind the disease are largely unknown. Objective: The present study aimed to find out the determinants of dysfunctional uterine bleeding. Materials and Methods: In this cross-sectional study 50 patients of dysfunctional uterine bleeding (DUB) were recruited from different tertiary hospitals in Dhaka city. Clinical parameters have been recorded using a predesigned questionnaire and analyzed using SPSS for Windows version 16.0 and Microsoft Office Excel. Results: In the studied patients, about 38\% were suffering from type 2 diabetes mellitus, $12 \%$ from obesity and 18\% from hypertension. Almost all the patients (96\%) were suffering from anemia. Histological findings have shown that endometrium of $63 \%$ patients were in proliferative phase, about $16 \%$ were in secretory phase and $11 \%$ patients were with cystic hyperplasia. Ultrasonographic results have shown that about $72 \%$ of the patients had bulky uterus. Ninety two percent patients carried more than two pregnancies, and $40 \%$ patients carried $\geqslant 5$ pregnancies. Conclusion: Bulky uterus and multiple pregnancies may be associated with dysfunctional uterine bleeding in Bangladeshi women.
\end{abstract}

Key words: Dysfunctional uterine bleeding; Bulky uterus; Multiparity

J Enam Med Col 2016; 6(1): 23-27

\section{Introduction}

Dysfunctional uterine bleeding (DUB) is defined as excessively heavy, prolonged or frequent bleeding of uterine origin that is not due to pregnancy or any recognizable pelvic or systemic disease. It is, therefore, a diagnosis of exclusion. The mechanisms for the abnormal bleeding and the site from which it arises are largely unknown. ${ }^{1}$ DUB occurs in 9 to 14 percent of women between menarche and menopause, significantly impacting quality of life and imposing financial burden. ${ }^{2}$ The etiologies and treatments for abnormal uterine bleeding over the reproductive years are best understood in the context of normal menstrual physiology. A normal cycle starts when pituitary follicle-stimulating hormone induces ovarian follicles to produce estrogen which stimulates proliferation of the endometrium. A luteinizing hormone surge prompts ovulation; the resultant corpus luteum produces progesterone, inducing a secretory endometrium. In the absence of pregnancy, estrogen and progesterone levels decline, and withdrawal bleeding occurs 13 to 15 days postovulation. ${ }^{3}$ Disruption of normal physiology, anatomic changes in the endometrium, or endometrial cancer may result in abnormal uterine bleeding. Genital bleeding during childhood, uterine bleeding that

1. Assistant Professor, Department of Gynecology and Obstetrics, Bangladesh Institute of Research and Rehabilitation in Diabetes, Endocrine and Metabolic Disorders-2 (BIRDEM-2) General Hospital, Dhaka

2. Senior Medical Officer, Department of Gynecology and Obstetrics, BIRDEM-2 General Hospital, Dhaka

3. Professor, Department of Gynecology and Obstetrics, BIRDEM-2 General Hospital, Dhaka

Correspondence Nahid Sultana, Phone: 01819-129110 
requires urgent intervention and postmenopausal uterine bleeding are also abnormal. Terms associated with abnormal uterine bleeding (AUB) are inconsistently defined in the literature, complicating the approach to evaluation and management. ${ }^{4}$ International experts are working to develop consensus on these definitions to improve evidence-based care. ${ }^{4}$ Dysfunctional uterine bleeding that occurs from adolescence through perimenopause can be broadly divided into two categories: anovulatory and ovulatory. Anovulatory bleeding is characterized by irregular or infrequent periods, with flow ranging from light to excessively heavy. ${ }^{5}$ Terms commonly associated with anovulatory bleeding include amenorrhea (absence of periods for more than three cycles), oligomenorrhea (menses occurring at intervals of more than 35 days), metrorrhagia (menses at irregular intervals with excessive bleeding or lasting more than seven days), and dysfunctional uterine bleeding (anovulatory bleeding in which underlying etiologies have been ruled out). ${ }^{3}$ Anovulatory cycles tend to be more common at puberty and after age 40, times at which irregular ovulation is often encountered. ${ }^{6}$ Although DUB is a common problem in Bangladesh and all over the world, determinants behind the disease have not been studied well. Therefore, the present study aimed to find out the determinants of DUB in Bangladeshi women.

\section{Materials and Methods}

In this cross-sectional study, 50 patients (25-55 years of age) suffering from DUB have been recruited from the Out-Patient Department (OPD) of different tertiary hospitals of Dhaka city.

Dysfunctional uterine bleeding has been diagnosed by exclusion. Seventy cases were recruited primarily on the basis of history and clinical examinations. Out of these, 20 patients were subsequently found to have organic pathology, diagnosed mainly from removed specimen after hysterectomy or histopathological examination of curettage and by ultrasonography. Cervical erosion and chronic cervicitis were not considered as organic pathology as these are present in many parous women in our country. Data of each patient have been recorded using a predesigned questionnaire and analyzed using SPSS for Windows version 16.0 and Microsoft Office Excel.

\section{Results}

In this study 50 patients with dysfunctional uterine bleeding (DUB) were recruited from different tertiary hospitals in Dhaka city. Maximum patients $(58 \%)$ were more than 40 years of age (Fig 1). Among the patients only $20 \%$ had monthly income more than Tk. 10000/= and 30\% had monthly income less than Tk. 5000/= (Fig 2).

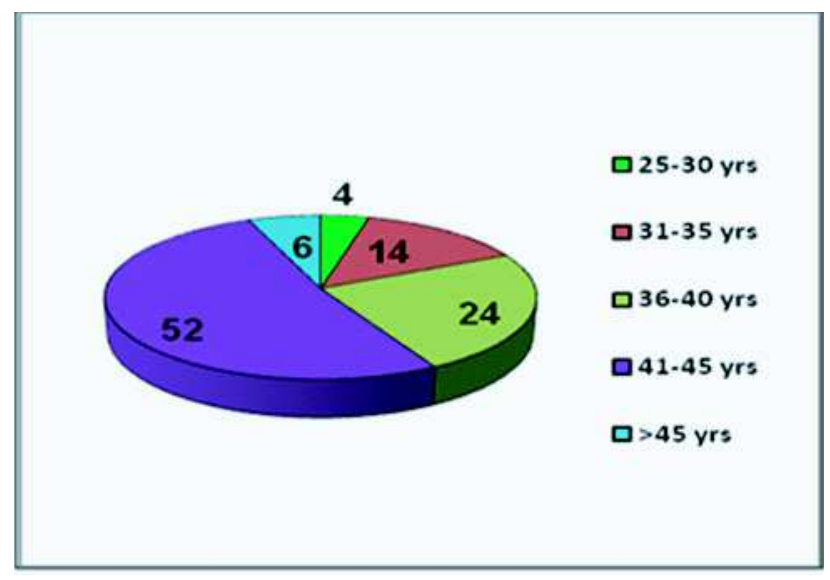

Fig 1. Distribution of patients (in percent) in different age groups

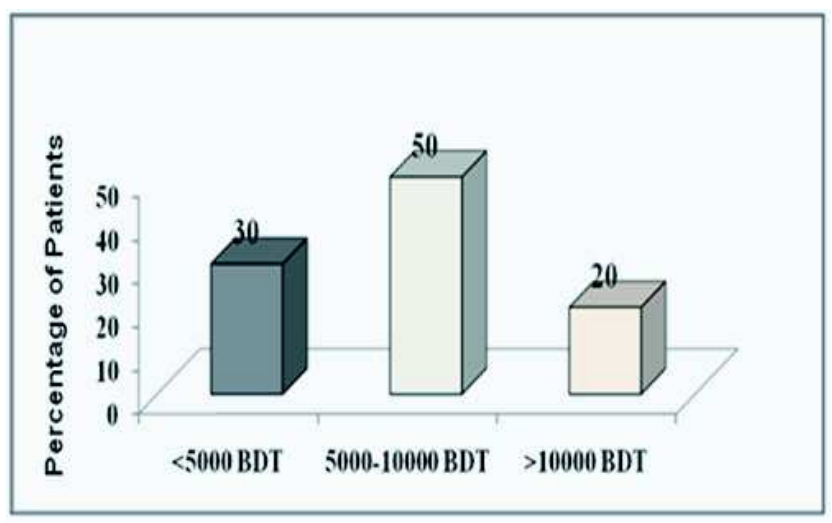

Fig 2. Monthly income (BDT) of the patients

After analysis of medical history it was found that $38 \%$ subjects were suffering from type 2 diabetes mellitus, $12 \%$ from obesity, $18 \%$ from hypertension, $10 \%$ from both diabetes and hypertension and $4 \%$ from hypothyroidism. Almost all the patients (96\%) were anemic (Fig 3).

About $60 \%$ of the patients had been suffering from DUB for more than one year and $80 \%$ had history of past abortion, among them 20 patients $(40 \%)$ had multiple abortions (data not shown in figure). 
About $60 \%$ patients had hemoglobin level less than $50 \%$ and only $20 \%$ patients had hemoglobin level more than $60 \%$ (Fig 4). Histological findings have shown that endometrium of $63 \%$ patients was in proliferative phase, $16 \%$ was in secretory phase and $11 \%$ patients had cystic hyperplasia (Fig 5). Ninety two percent patients carried more than two pregnancies and $40 \%$ patients carried $\geqslant 5$ pregnancies (Fig 6).

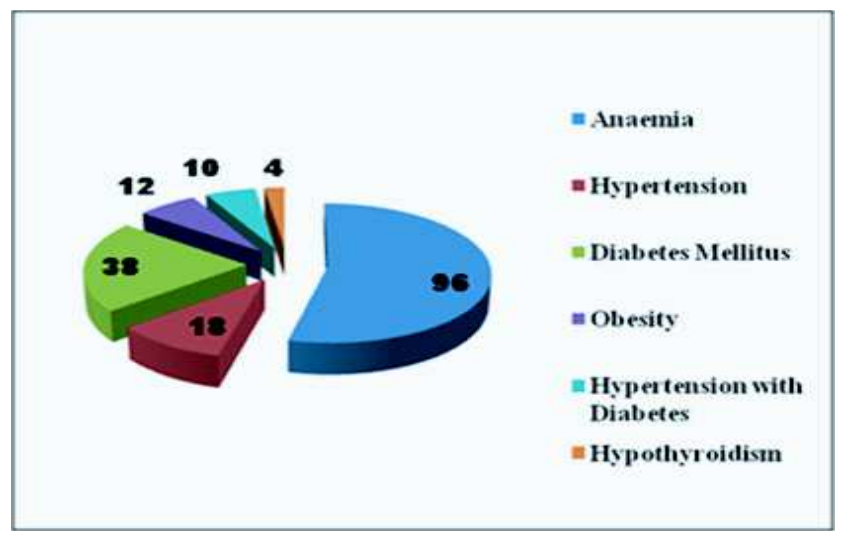

Fig 3. Distribution of patients according to medical history

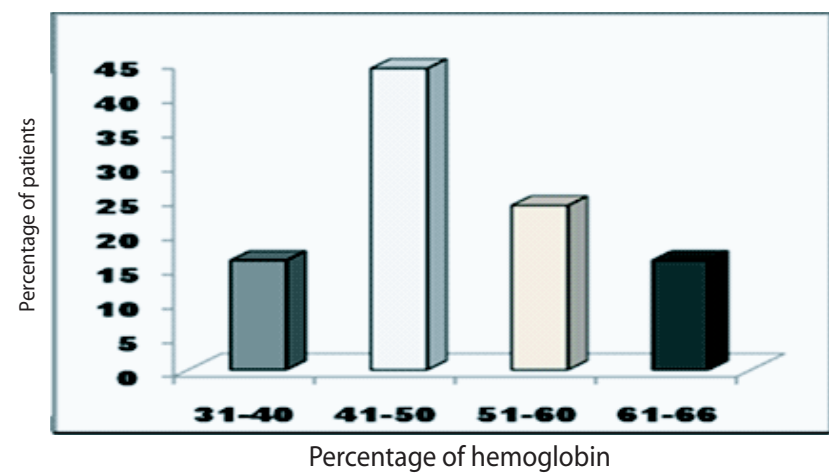

Fig 4. Distribution of patients according to hemoglobin status

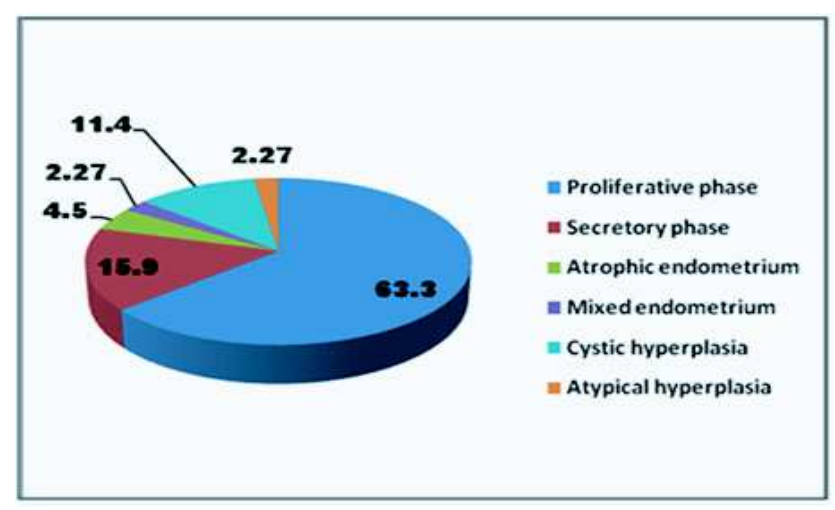

Fig 5. Distribution of subjects (in percent) according to histopathology of endometrium

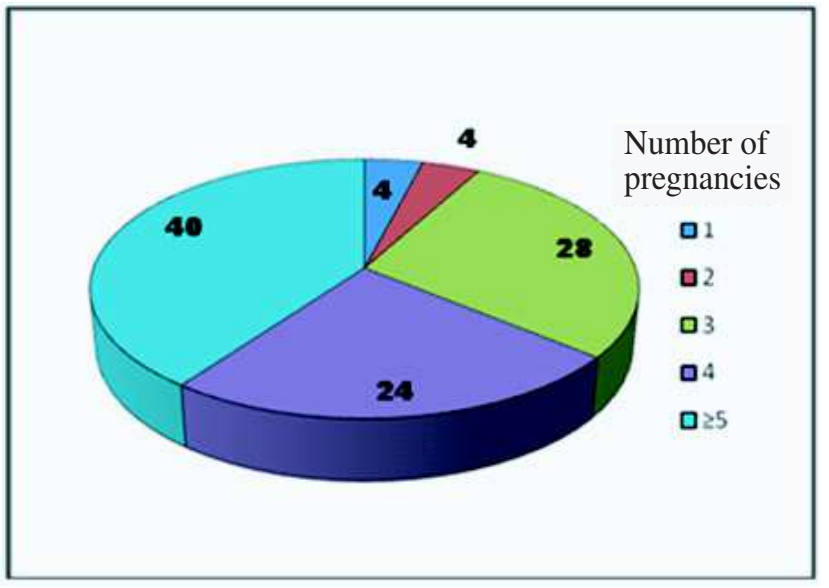

Fig 6. Distribution of subjects (in percent) according to number of pregnancies

Ultrasonographic study has shown that about $72 \%$ of the patients had bulky uterus. Endometrium of $20 \%$ patients was thickened and cystic ovary was found in $18 \%$ patients (Table I). DUB patients responded well to tranexamic acid and combination of estrogen and progesterone compared to single drug progesterone (Table II).

Table I: Ultrasonographic findings of uterus, endometrium and ovary in DUB patients

\begin{tabular}{|l|l|c|}
\hline \multicolumn{2}{|c|}{ Ultrasonographic findings } & Frequency (\%) \\
\hline \multirow{3}{*}{ Size of Uterus } & Normal & $14(28)$ \\
\cline { 2 - 3 } Endometrium & Bulky & $36(72)$ \\
\hline \multirow{3}{*}{ Ovary } & Normal & $38(76)$ \\
\hline & Thickening & $10(20)$ \\
\cline { 2 - 3 } & Thinning & $2(4)$ \\
\hline & Normal & $41(82)$ \\
\hline
\end{tabular}

Table II: Mode of treatment and outcomes of the DUB patients

\begin{tabular}{|c|c|c|c|c|c|c|}
\hline \multicolumn{3}{|c|}{ Medical treatment $(\mathrm{n}=40)$} & \multicolumn{4}{c|}{ Curettage (n=21) } \\
\hline Mode & Number & $\begin{array}{c}\text { Responded } \\
\text { to drug }\end{array}$ & $\begin{array}{c}\text { Immediate } \\
\text { response }\end{array}$ & Late response \\
\hline Progesterone & 24 & 12 & $\begin{array}{c}\text { Good } \\
\mathrm{n}(\%)\end{array}$ & $\begin{array}{c}\text { Poor } \\
\mathrm{n}(\%)\end{array}$ & $\begin{array}{c}\text { Good } \\
\mathrm{n}(\%)\end{array}$ & $\begin{array}{c}\text { Poor } \\
\mathrm{n}(\%)\end{array}$ \\
\hline $\begin{array}{l}\text { Oestrogen \& } \\
\text { progesterone }\end{array}$ & 6 & 6 & $19(90.5)$ & $2(9.5)$ & $15(71.4)$ & $6(28.6)$ \\
\hline Tranexamic acid & 10 & 8 & & & & \\
\hline
\end{tabular}

\section{Discussion}

Dysfunctional uterine bleeding (DUB) that adversely affects women is a major health problem. The term 
DUB is used to cover all forms of abnormal bleeding for which an organic cause cannot be found and used differently in different countries. In USA, DUB refers to anovulatory bleeding, signifying a hormonal imbalance while ovulatory DUB is referred to as 'menorrhagia' signifying a structural lesion or a bleeding disorder. ${ }^{7}$ The lack of uniformity makes it a poorly defined diagnosis of exclusion. However, as it is a diagnosis of exclusion, it may be useful as a working clinical diagnosis and some consistency should be sought. European Society for Human Reproduction and Embryology defines DUB as 'excessive uterine bleeding (excessively heavy, prolonged or frequent) which is not due to demonstrable pelvic disease, complications of pregnancy or systemic disease'. This definition of DUB includes both ovulatory and anovulatory bleeding and excludes abnormal bleedings such as those that occur during hormonal replacement or oral contraceptives. ${ }^{7}$

DUB is a common problem in women in $30-50$ years age group. ${ }^{8}$ The pathophysiology is not fully understood and it is complex. DUB is a diagnosis of exclusion. Uterine and systemic causes must be excluded before the diagnosis is made. Ovulation occurs in about $80 \%$ of women with DUB. DUB also occurs at the extremes of the reproductive years; in the adolescent, coagulopathies should be excluded. Management is initially with medical treatments. Surgical procedures are performed only if other treatments prove ineffective. ${ }^{8}$

Although heavy menstrual bleeding is clinically defined as greater than $80 \mathrm{~mL}$ per cycle, less than half of women seeking medical attention for their bleeding lose more than this defined amount. ${ }^{9}$ This means that something other than the amount of bleeding is driving women to seek medical attention. Quality of life (QOL) is likely one factor influencing women with abnormal uterine bleeding (AUB) to request treatment. ${ }^{9}$

In clinical managements, non-steroidal antiinflammatory drugs (NSAIDs) or anti-prostaglandins reduce prostaglandin levels which are excessive in heavy menstrual bleeding, but the mechanism of action is not fully understood. Blood flow is reduced by about $30 \%$ and menstrual pain is also reduced. All the major NSAIDs available have been shown to be effective. Tranexamic acid (cyklokapron) is an antifibrinolytic agent that reduces the endometrial fibrinolytic enzymes that are increased in DUB. Menstrual blood loss is reduced by $45-60 \%$. Tranexamic acid is prescribed in only the heavy days of the menses, usually during the first 5 days. Tranexamic acid is the appropriate first line therapy for ovulatory DUB. ${ }^{10}$ The levonorgestrel (LNG) releasing intrauterine device (IUD) releases LNG at a low dose of $20 \mu \mathrm{g}$ per day leading to endometrial atrophy and thickened cervical mucus. There is a major reduction in blood loss (up to 97\%) after 12 months of use. The IUD is suitable in both ovulatory and anovulatory DUB. The IUD is a contraceptive and its duration of action is 5 years. ${ }^{10}$ The progestogens, or progestins, are usually used cyclically but can be used continuously. These are the first line treatment in anovulatory DUB and are prescribed in the luteal phase from about day 15 to day 25 . In ovulatory DUB, the progestogen is prescribed from day 5 to day 25. Danazol has also been found to effectively reduce heavy menstrual bleeding but its use has been limited because of its recommended short term use and side effects including headaches. ${ }^{10}$

In our study it has been shown that 12 out of 24 DUB patients responded well with progesterone treatment whereas all the patients ( 6 out of 6 ) have responded well when we have used oestrogen and progesterone combined. On the other hand, eight out of 10 DUB patients responded well with tranexamic acid treatment.

The clinical management of women with DUB aims to improve the patient's symptoms and quality of life. There are dearths of information regarding the factors for the development of DUB. In the studied subjects, 24 percent are between 36 to 40 years of age and 52 percent are between 41 to 45 years of age. Although 30 percent of the studied DUB patients are in low income group but about half of the patients are from middle income group and only 20 percent are from higher income group. Since DUB is associated with heavy blood loss, almost all the patients (96\%) suffered from anemia. In the studied DUB patients $18 \%$ was suffering from hypertension, $12 \%$ from obesity and $38 \%$ from diabetes mellitus. Because of the anemia patients' immunity has decreased, which may invite other diseases.

It has been reported earlier that obesity is associated with abnormal uterine bleeding. ${ }^{11}$ There is clear association between obesity, endometrial polyps, endometrial hyperplasia and ovulatory dysfunction. As PCOs are associated with obesity and obesity augments its development, many of the effects of obesity on 
menstrual disorders are manifested through PCOs. A survey of pre-menopausal women with endometrial polyps found that $82 \%$ reported abnormal uterine bleeding. In obese women particularly in combination with hypertension, there is an increased risk for development of polyp. ${ }^{11}$

Although we have not compared our results with healthy control subjects but our results documented that about $76 \%$ of the patients have presented with bulky uterus which may be an important determinant for this disease. Each pregnancy is a stressful condition for women. In our study more than $90 \%$ of the studied DUB patients were with more than two children. So, it may also be an important determinant for this disease condition. Therefore, it may be concluded that bulky uterus and multiple pregnancies may be associated with dysfunctional uteruine bleeding in Bangladeshi women.

\section{Acknowledgement}

We are grateful to Dr Md Omar Faruque, Associate Professor, Department of Physiology and Molecular Biology, Bangladesh University of Health Sciences, Dhaka for his advice to prepare this article.

\section{References}

1. Farrell E, Frcog F. Dysfunctional uterine bleeding. Aust Family Physician 2004; 33(11): 906-908.

2. Fraser IS, Langham S, Uhl-Hochgraeber K. Health-related quality of life and economic burden of abnormal uterine bleeding. Expert Rev Obstet Gynecol 2009; 4(2): 179-189.

3. Sweet MG, Schmidt-Dalton TA, Weiss PM. Evaulation and management of abnormal uterine bleeding in premenopausal women. Am Fam Physician 2012; 85(1): $35-43$.

4. Fraser IS, Critchley HO, Munro MG, Broder M. A process designed to lead to international agreement on terminologies and definitions used to describe abnormalities of menstrual bleeding. Fertil Steril 2007; 87(3): 466-476. [Published correction appears in Fertil Steril 2007; 88(2): 538].

5. ACOG Committee on Practice Bulletins-Gynecology. American College of Obstetricians and Gynecologists. ACOG practice bulletin: management of anovulatory bleeding. Int J Gynaecol Obstet 2001; 72(3): 263-271.

6. Chen BH, Giudice LC. Dysfunctional uterine bleeding. West J Med 1998; 169: 280-284.

7. Bunkheila AK, Powell MC. Menorrhagia and dysfunctional uterine bleeding. Current Obs Gynaecol 2002; 12: 328-333.

8. Bongers MY, Mol BW, Brolmann HA. Current treatment of dysfunctional uterine bleeding. Maturitas 2004; 47: 159-174.

9. Matteson KA, Boardman LA, Munro MG, Clark MA. Abnormal uterine bleeding: a review of patient based outcome measures. Fertil Steril 2009; 92: 205-216.

10. Guidelines for the management of heavy menstrual bleeding. National Health Committee New Zealand, 1998.

11. Bano R, Datta S, Mahmood TA. Heavy menstrual bleeding. Obstetrics, Gynaecology and Reproductive Medicine 2013; 24: 1 . 